\title{
The CREST reactive-burn model for explosives
}

\author{
C. Handley ${ }^{\mathrm{a}}$, N. Whitworth, H. James, B. Lambourn, and M-A. Maheswaran
}

AWE, Aldermaston, Reading, Berkshire, RG7 4PR, UK

\begin{abstract}
CREST is an innovative reactive-burn model that has been developed at AWE for simulating shock initiation and detonation propagation behaviour in explosives. The model has a different basis from other reactive-burn models in that its reaction rate is independent of local flow variables behind the shock wave e.g. pressure and temperature. The foundation for CREST, based on a detailed analysis of data from particle-velocity gauge experiments, is that the reaction rate depends only on the local shock strength and the time since the shock passed. Since a measure of shock strength is the entropy of the non-reacted explosive, which remains constant behind a shock, CREST uses an entropydependent reaction rate. This paper will provide an overview of the CREST model and its predictive capability. In particular, it will be shown that the model can predict a wide range of experimental phenomena for both shock initiation (e.g. the effects of porosity and initial temperature on sustained-shock and thin-flyer initiation) and detonation propagation (e.g. the diameter effect curve and detonation failure cones) using a single set of coefficients.
\end{abstract}

\section{Introduction}

CREST is an innovative reactive-burn model for plastic bonded explosives, that uses entropy-dependent reaction rates. A detailed analysis of gas-gun particle-velocity gauge data [1] suggests that, to first order, reaction rates depend only on the shock strength and the time since the shock passed. In CREST, a function of the entropy of the non-reacted explosive with units of specific internal energy, known here as "entropy", is used to represent shock strength. The entropy is calculated from the finite-strain non-reacted equation of state, in conjunction with the snow-plough porosity model. The reaction-rate equations are formulated to incorporate the effect of time and are given in approximate form below. See references [2] and [3] for details.

$$
\begin{gathered}
\dot{\lambda}=m_{1} \dot{\lambda}_{1}+m_{2} \dot{\lambda}_{2}+m_{3} \dot{\lambda}_{3} \\
\dot{\lambda}_{1}=b_{1} t\left(1-\lambda_{1}\right) \\
\dot{\lambda}_{2}=b_{2} t \lambda_{1}\left(1-\lambda_{2}\right) \\
\dot{\lambda}_{3}=b_{3} t \lambda_{1}\left(1-\lambda_{3}\right)
\end{gathered}
$$

The CREST weighting factors $m_{1}, m_{2}$ and $m_{3}$, and parameters $b_{1}, b_{2}$ and $b_{3}$ which control the time to peak reaction rate, are functions of the entropy. They are tuned to gas-gun particle-velocity gauge traces, the Pop-plot and the diameter effect curve. Once chosen, these coefficients are used without alteration to investigate a wide range of experimental phenomena.

\footnotetext{
a e-mail: caroline.handley@awe.co.uk
}

This is an Open Access article distributed under the terms of the Creative Commons Attribution-Noncommercial License 3.0, which permits unrestricted use, distribution, and reproduction in any noncommercial medium, provided the original work is properly cited. 

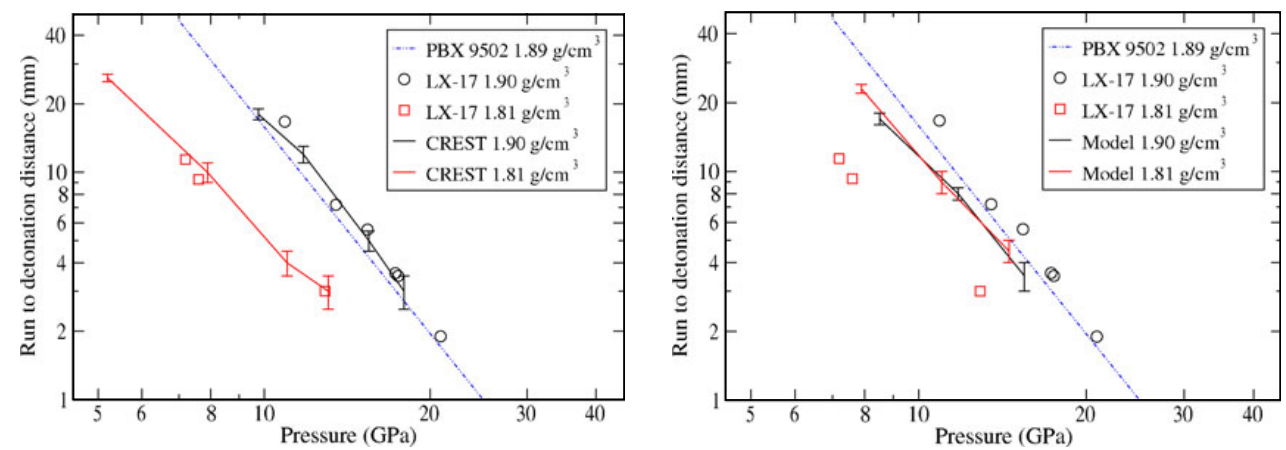

Fig. 1. Prediction of the Pop-plot for LX-17 [7] using the entropy-based CREST model (left) and a similar, pressure-based model (right).

This paper will give an overview of the capabilities of the CREST model for PBX 9502, a TATBbased explosive. Originally published in 2007 and tuned only to one-dimensional gas-gun data, this model was found to be capable of predicting thin-pulse and initiation threshold data [4]. Although it represented detonation failure behaviour in PBX 9502 rate sticks with remarkable accuracy, the calculated diameter effect curve did not capture the upturn observed in the experimental data at large charge sizes [5]. To improve the model, it was found to be necessary to include a third, slow reaction rate [3] whose coefficients are tuned to match the diameter effect curve. The CREST coefficients for PBX 9502 in [3] are used for all the simulations in this paper.

\section{Shock initiation}

The CREST reaction rate was tuned to PBX 9502 experimental data at ambient temperature $\left(23^{\circ} \mathrm{C}\right)$ and a single density $\left(1.89 \mathrm{~g} / \mathrm{cm}^{3}\right)$. One of the advantages of CREST is that the non-reacted equation of state allows the initial density (or porosity) of the explosive and the initial temperature to be dialled in. The entropy and pressure behind a shock are then automatically adjusted to account for the change in porosity or temperature. In this section, CREST will be used to predict the sensitivity of shock initiation data to changes in the initial porosity and temperature of PBX 9502.

\subsection{Predicting the effect of porosity [3]}

Pop-plot data is available for PBX 9502, which comprises 95\% TATB and 5\% Kel-F binder, but only over a narrow density range [6]. LX-17 is a similar explosive, which comprises $92.5 \%$ TATB and 7.5\% Kel-F, and Pop-plot data is available for a range of porosities up to 7\% [7]. The CREST model was applied to the LX-17 experiments simply by changing the initial density of the explosive. No other modifications were made and, in particular, the reaction-rate coefficients were not changed. Figure 1 shows that CREST, with its entropy-dependent reaction rate, correctly predicts the change in the Popplot as the density is reduced from 1.90 to $1.81 \mathrm{~g} / \mathrm{cm}^{3}$.

Results from a pressure-based model that uses the same equations of state and reaction-rate equations, but with parameters $m_{1}, m_{2}, m_{3}, b_{1}, b_{2}$ and $b_{3}$ depending on pressure rather than entropy, are also shown in figure 1 . The pressure-based model is unable to predict the effect of porosity; the Pop-plot hardly moves as the density is reduced from 1.90 to $1.81 \mathrm{~g} / \mathrm{cm}^{3}$. This is because, when the density is reduced from theoretical maximum density to $1.80 \mathrm{~g} / \mathrm{cm}^{3}$, the entropy of shocked non-reacted PBX 9502 (impacted by Kel-F at $2.5 \mathrm{~km} / \mathrm{s}$ ) increases by $85 \%$, while the pressure decreases by $11 \%$. However, the reaction growth and therefore the Pop-plot of an explosive is a function not only of the reaction rate but also on the hydrodynamic catch-up factor $c /\left(U_{s}-u_{p}\right)$. As the density is reduced from TMD to $1.8 \mathrm{~g} / \mathrm{cm}^{3}$, the catch-up factor increases by $7 \%$. For an entropy-dependent model, the effect of the $85 \%$ increase in entropy and the $7 \%$ increase in the catch-up factor combine to produce a shift in Pop-plot, 

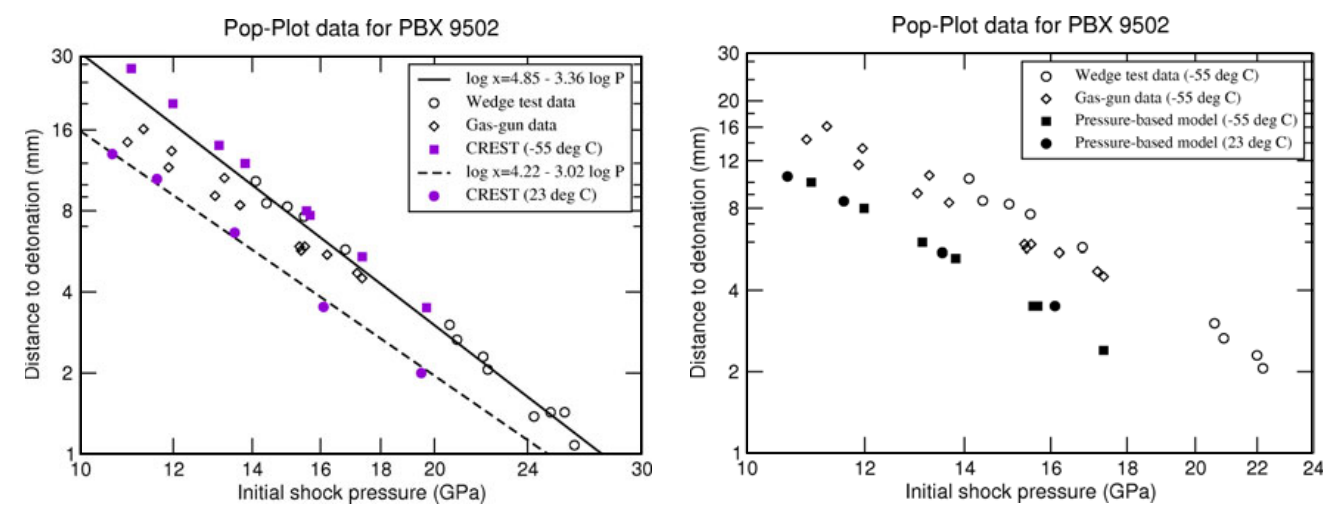

Fig. 2. Prediction of the Pop-plot for PBX 9502 cooled to $-55^{\circ} \mathrm{C}[9]$ using the entropy-based CREST model (left) and a similar, pressure-based model (right).

in agreement with the experimental data. In contrast, for a pressure-dependent model the effect of the $11 \%$ reduction in pressure and the $7 \%$ increase in catch-up factor approximately cancel out, which explains why the Pop-plot hardly moves as the porosity is varied. We believe that this is a fundamental limitation of pressure-dependent reactive-burn models.

\subsection{Predicting the effect of temperature [8]}

Pop-plot data for PBX 9502 is available at ambient initial temperature (assumed to be $23^{\circ} \mathrm{C}$ ), cold at $-55^{\circ} \mathrm{C}$ and hot at $+75^{\circ} \mathrm{C}$ [9], although there is only space to illustrate the cold data here. The CREST model for PBX 9502 was used to predict this data, by modifying only the initial specific internal energy and density of the explosive to give the correct starting temperature. No changes were made to the equation of state or reaction-rate coefficients.

Figure 2 shows that CREST correctly predicts the trend in the experimental data i.e. that the cold explosive is significantly less sensitive than the ambient PBX 9502. In contrast, a similar model with a pressure-dependent reaction rate produces only a very small change in the Pop-plot when going from ambient to $-55^{\circ} \mathrm{C}$, because the change in shock pressure with initial temperature is much less than the corresponding change in entropy. Therefore, entropy-dependent reactive-burn models like CREST have two significant advantages over pressure-based models, in predicting the effects of both initial density and temperature on the sensitivity of explosives.

\subsection{Predicting the initiation threshold}

CREST has previously [4] been used to model one-dimensional shock initiation threshold experiments on RX-03-AU [10], which has the same composition as PBX 9502, at three different densities. Recently, Maheswaran has repeated the thin-flyer "go" and "no go" simulations using the latest CREST model [3] and her results are illustrated in Fig. 3. She has also modelled two-dimensional initiation threshold data [11], where the flyer is both thin and narrow, and these results will be published at a later date.

Figure 3 shows that, for a given density, CREST is able to predict the increase in mylar flyervelocity that is needed to initiate the explosive as the flyer-thickness is reduced [10], although agreement is not perfect for the very thinnest flyers. The model also predicts the increase in sensitivity (lower flyer-velocities are needed to initiate the explosive for a given flyer thickness) as the initial density is reduced. This is consistent with the observations in section 2.3 that CREST is able to predict the effect of initial density (or porosity) of the explosive on its sensitivity. 


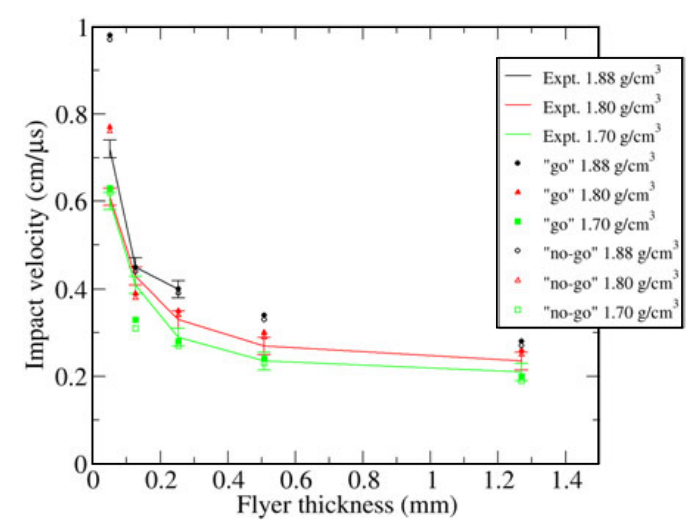

Fig. 3. Prediction of 1D initiation threshold data for PBX 9502 [10] using CREST (dots).

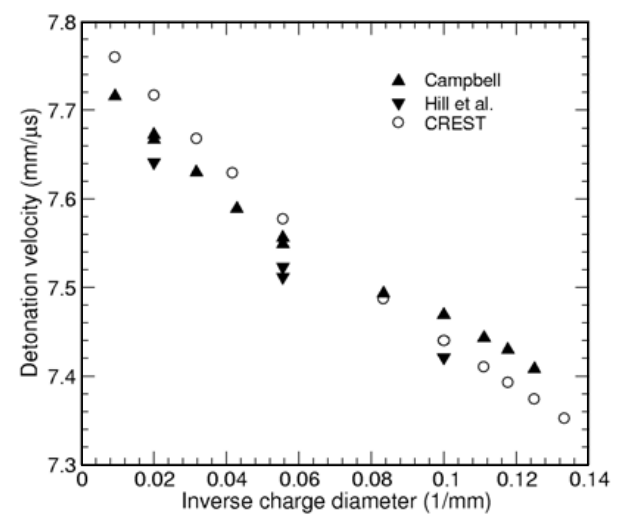

Fig. 4. Prediction of diameter effect curve for PBX9502 [13,14] using CREST.

\section{Detonation propagation}

The CREST reactive-burn model can also be used to simulate detonation propagation experiments. Whitworth [5] showed that, although the earlier CREST model could predict detonation failure behaviour, a third slow reaction was needed to accurately simulate the diameter effect curve at large charge sizes. In this section, Lambourn's improved reaction rate [3] is used to simulate rate stick and failure cone experiments.

\subsection{Diameter effect curve [12]}

Rate-stick experiments on PBX 9502 at three initial temperatures (ambient, $-55^{\circ} \mathrm{C}$ and $+75^{\circ} \mathrm{C}$ ) have provided the diameter effect curve, the failure diameter and wave-shape data $[13,14]$. CREST has been used to model all these effects, but there is only space to show the diameter effect results here, in Fig. 4. Overall, CREST accurately models the entire diameter effect curve although the predicted failure diameter is $\sim 2.0 \mathrm{~mm}$ smaller than observed experimentally. This suggests that improvements need to be made to the third, slow reaction rate.

\subsection{Detonation failure cones [12]}

As Hill has pointed out [15], dynamic detonation failure experiments are a challenging test for any reactive-burn model. Dynamic failure data is available from PBX 9502 failure cone experiments, with 
New Models and Hydrocodes for Shock Wave Processes in Condensed Matter

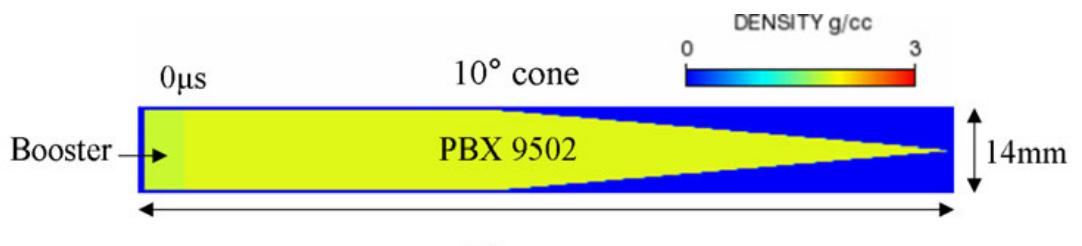

$130 \mathrm{~mm}$

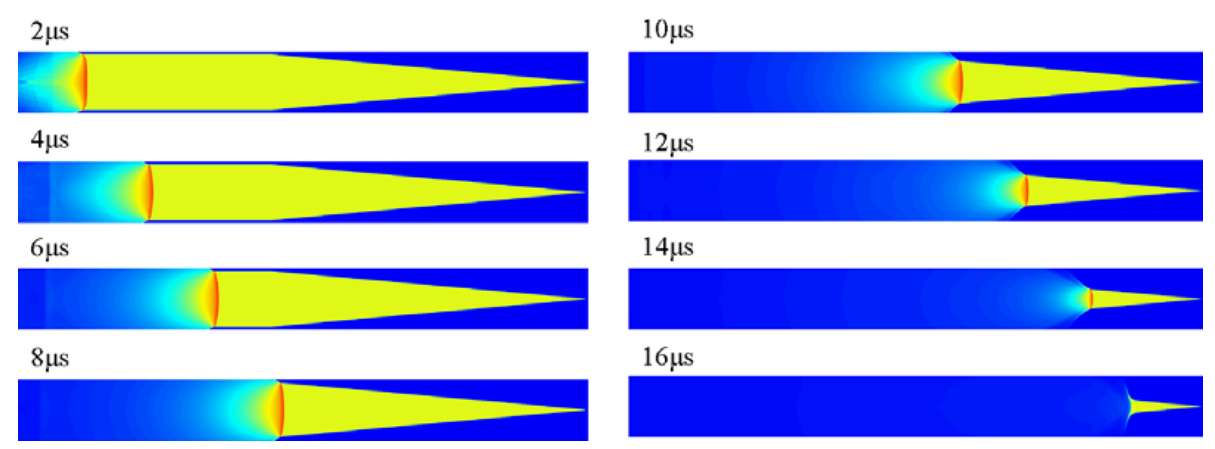

Fig. 5. Density plot from CREST simulation of a PBX 9502 failure cone.

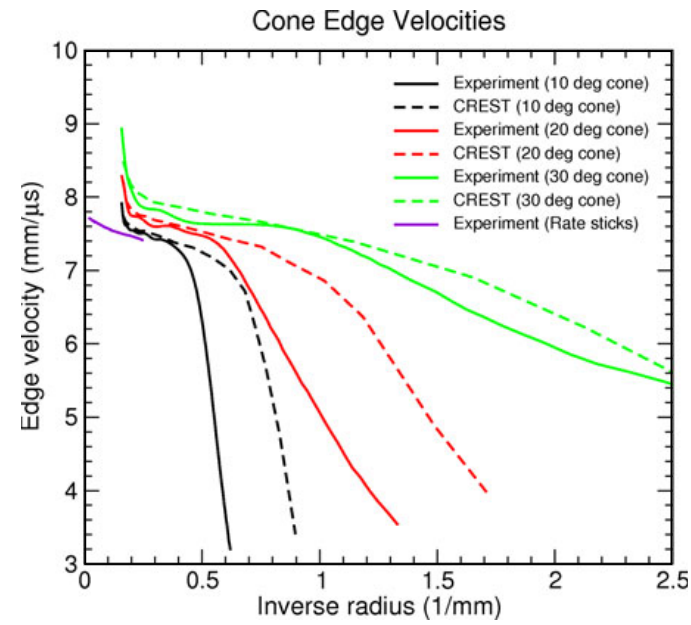

Fig. 6. Predictions of PBX 9502 failure-cone edge velocities using CREST.

tip angles between 10 and $90^{\circ}$ [15]. Snapshots from a CREST simulation of one of these experiments are given in Fig. 5. The failure cone is initiated using a booster with a single detonation point. Between 2 and $8 \mu \mathrm{s}$, the detonation wave propagates along the cylinder of PBX 9502, until steady state is achieved. After $8 \mu \mathrm{s}$, the detonation wave passes into the cone and begins to slow down as the charge radius shrinks. The detonation wave has failed by $16 \mu \mathrm{s}$, leaving a tip of non-reacted PBX 9502 that closely resembles proton radiographs from similar failure cone experiments [16].

Figure 6 shows a quantitative comparison of the failure-cone edge velocity data. The CREST simulations agree with the experiments [15], although failure occurs at smaller radii than observed in the experiments. 


\section{Conclusion}

The examples in this paper show that the CREST model for PBX 9502 can predict a wide range of experimental phenomena using one set of equation of state and reaction-rate coefficients. By its use of a porosity model, the non-reacted equation of state automatically adjusts the shock entropy and pressure to account for changes to the initial temperature or porosity of the explosive. This allows CREST to predict the change in sensitivity of explosives as their initial temperature and/or density is varied. In contrast, it has been demonstrated that a pressure-based model that uses the same equations of state is unable to simulate this effect. We believe that this is a fundamental limitation of pressure-based reactive-burn models. CREST is also able to model detonation propagation and failure experiments, although an under-prediction of the failure diameter suggests that the third, slow reaction rate needs to be improved.

\section{References}

1. H.R. James, B.D. Lambourn, Journal of Applied Physics, 100, 084906 (2006)

2. C.A. Handley, Proceedings of the $13^{\text {th }}$ International Detonation Symposium, 864-870 (2006)

3. C.A. Handley, B.D. Lambourn, Shock Compression of Condensed Matter, 221-224 (2009)

4. C.A. Handley, Shock Compression of Condensed Matter, 373-376 (2007)

5. N.J. Whitworth, Shock Compression of Condensed Matter, 881-884 (2007)

6. R.L. Gustavsen, S.A. Sheffield, R.R. Alcon, Journal of Applied Physics, 99, 114907 (2006)

7. R.K. Jackson et al., Proceedings of the $6^{\text {th }}$ Symposium (Int.) on Detonation, 755-765 (1976)

8. N.J. Whitworth, B.D. Lambourn, Shock Compression of Condensed Matter, 458-461 (2009)

9. R.L. Gustavsen et al., Proceedings of the $13^{\text {th }}$ International Detonation Symposium, 970-979 (2006), and R.L. Gustavsen et al., Private Communication (2009)

10. C.A. Honodel et al., Proceedings of the $7^{\text {th }}$ Symposium (Int.) on Detonation, 425-434 (1981)

11. R.K. Jackson et al., Proceedings of the $6^{\text {th }}$ Symposium (Int.) on Detonation, 755-765 (1976)

12. N.J. Whitworth, C.A. Handley, B.D. Lambourn, to appear in Proceedings of the $14^{\text {th }}$ International Detonation Symposium, (2010)

13. A.W. Campbell, Propellants, Explosives, Pyrotechnics, 9, 183-187 (1984)

14. L.G. Hill, J.B. Bdzil and T.D. Aslam, Proceedings of the $11^{\text {th }}$ International Detonation Symposium, 1029-1037 (1998)

15. T.A. Salyer, L.G. Hill, Proceedings of the $13^{\text {th }}$ Int. Detonation Symposium, 24-34 (2006)

16. E.N. Ferm et al., Proceedings of the $13^{\text {th }}$ International Detonation Symposium, 970-979 (2006)

(c) British Crown Owned Copyright 2010/MOD 